Research Article

Plant Genetics

\title{
Functional analysis of 4-coumarate: CoA ligase from Dryopteris fragrans in transgenic tobacco enhances lignin and flavonoids
}

\author{
Shan-Shan $\mathrm{Li}^{1,2}$ (D), Ying Chang ${ }^{3}$, Bo Li ${ }^{1,2}$, Shu-Li Shao ${ }^{1,2}$ and Zhen-Zhu-Zhang ${ }^{1,2}$ \\ ${ }^{1}$ Qiqihar University, College of Life Sciences and Agriculture and Forestry, Qiqihar, China, \\ ${ }^{2}$ Heilongjiang Provincial Key Laboratory of Resistance Gene Engineering and Protection of Biodiversity in \\ Cold Areas, Qiqihar, China. \\ ${ }^{3}$ Northeast Agricultural University Laboratory of Plant Research College of Life sciences, Harbin, China.
}

\begin{abstract}
4-Coumaric acid: coenzyme A ligase (4CL) is a key enzyme in the phenylpropanoid metabolic pathway that regulates the biosynthesis of lignin and flavonoids. Therefore, the study of $4 \mathrm{CL}$ is important to explore the accumulation and regulation of metabolites. This study investigated the role that the $4 C L 2$ gene from Dryopteris fragrans (Df4CL2) plays in the metabolite synthesis. Changes in gene expression, enzyme activity, and the content of lignin and flavonoids were measured in different tissues of tobacco as model plant that was successfully transferred with DF4CL2. Tobacco plants with Df4CL2 (transgenic tobacco, TT) were successfully obtained via the Agrobacteriumtransformation method. This TT tended to be thicker and had an earlier flowering period than wild type tobacco (WT). The expression levels of Df4CL2 were higher in the stem, leaf, and root in TT compared to WT. In addition, compared to WT, TT had higher $4 \mathrm{CL}$ enzyme activity and higher lignin and flavonoids contents. This suggests that Df4CL2 is involved in the synthesis of lignin and flavonoids in $D$. fragrans. This research provides important evidence toward understanding the phenylpropanoid metabolic pathway in ferns.
\end{abstract}

Keywords: phenylpropanoid metabolic pathway, 4-coumaric acid: coenzyme A ligase, ferns, Dryopteris fragrans, metabolites.

Received: November 30, 2018; Accepted: July 12, 2019.

\section{Introduction}

The secondary metabolic pathways of phenylpropanoid have been studied in detail, especially in angiosperms, where the metabolic pathway has been explored in detail (Dixon et al., 2002; Hamberger and Hahlbrock, 2004). In this particular metabolic pathway, 4-Coumaric acid: coenzyme A ligase (4CL; EC 6.2.1.12) is a key enzyme, located in the transition from the general pathway to the downstream branch pathway (Vassão et al., 2010; Gui et al., 2011). 4CL uses cinnamic acid, 4-coumaric acid, 5-hydroxyferic acid, sinapic acid, caffeic acid, and ferulic acid as substrates to form the corresponding acyl-CoA thioester in the presence of ATP and $\mathrm{Mg}^{2+}$. These become substrates in different downstream branch pathways. 4CL generally exists as an isozyme in different plants and has different substrate preferences. It regulates the amount of CoA esters by catalyzing different substrates, or it enters the lignin biosynthesis-specific pathway through a reaction catalyzed by cinnamoyl-CoA reductase (CCR) and cinnamyl alcohol dehydrogenase (CAD). 4CL can also enter flavonoids

Send correspondence to Shan-Shan Li. Qiqihar University, College of Life Sciences and Agriculture and Forestry, Qiqihar, 161006, China. E-mail: lishanshan83@163.com. through either chalcone synthase (CHS) or chalcone isomerase (CHI) (Harding et al., 2002). Recent studies indicated that $4 \mathrm{CL}$ is one of the rate-limiting enzymes in the phytochemical monomer synthesis pathway of vascular plants. This has also become a focus in genetic engineering research to regulate the lignin content of vascular plants (Emiliani et al., 2009; Rao et al., 2015; Lavhale et al., 2018; Liu et al., 2018). Therefore, the in-depth study of the $4 C L$ gene and enzyme activity not only has an important role in the process of plant growth and development, as well as in growth in adverse environments, but it also provides an important guidance in the production and regulation of secondary metabolites (Blach-Olszewska et al., 2008). 4CL has been studied in angiosperms, gymnosperms, and bryophytes; however, in ferns, its metabolic pathway and function remains not clearly identified.

Dryopteris fragrans is a perennial medicinal fern that is widely distributed in the Heilongjiang Province of China, especially in the magma gap around the volcanos in the Wudalianchi Scenic Area. The unique characteristics of this growth environment confer unique medicinal value on $D$. fragrans. As a folk medicine, D. fragrans is mainly used to treat arthritis and various skin diseases, including psoriasis, acne, rash, and dermatitis. Chinese and foreign re- 
searchers found that $D$. fragrans also has multiple features including deworming, antibacterial (Shen et al., 2005; Zhang et al., 2008a), antioxidant (Zhang et al., 2008b; Li et al., 2012a), analgesic (Wang et al., 2008), and antitumor (Ito et al., 2000; Li et al., 2012b) activities. A major class of functional substances contained in D. fragrans are flavonoids. The metabolic pathways and key enzymes resulting from the synthesis of compounds have become a major breakthrough in regulating these effective substances. The synthetic pathway of metabolites in ferns has not yet been fully understood, and the function of key enzymes remains unknown. Therefore, it is important to explore the synthesis and regulation of both flavonoids and lignin in metabolites from the genetic perspective.

In this study, the Df4CL2 gene of D. fragrans was transferred into the tobacco model plant, and the relationship between 4CL2, flavonoids, and lignin was identified by examining the genetic expression, enzyme activity, and metabolites. In doing so, a basis was provided for the identification of the phenylpropanoid metabolic pathway of this fern and for the extensive application of this species.

\section{Material and Methods}

\section{Genetic transformation of the gene}

The Df4CL2 (KF836752) gene obtained from prior experiments was used to construct the pBI121-Df4CL2 expression vector (Li et al., 2015). The genetic transformation of tobacco was performed through the Agrobacterium-mediated transformation as described by Hoekema et al. (1983). $\mathrm{T}_{1}$-generation-regenerated kanamycin-resistant plants were identified using PCR, and successfully identified plants were reserved for subsequent experiments. The specific primers for PCR identification were: 4CL-F, 5'-GGCTGAGGTCCTTCCCTCCTCTG-3', 4CL-R,5'ACTAGCGCTATTTGATTTTCTTAATGC-3'. PCR parameters were: initial denaturation for $5 \mathrm{~min}$ at $95^{\circ} \mathrm{C}$, followed by 35 cycles of denaturation for $30 \mathrm{~s}$ at $95{ }^{\circ} \mathrm{C}$, annealing for $30 \mathrm{~s}$ at $58{ }^{\circ} \mathrm{C}$, and extension at $72{ }^{\circ} \mathrm{C}$ for 2 min, with a final extension step for $10 \mathrm{~min}$ at $72{ }^{\circ} \mathrm{C}$. The PCR products were observed via $1 \%$ agarose gel electrophoresis (Li et al., 2015).

\section{Expression of Df4CL2 in transgenic tobacco}

RNA from root, stem, leaf, flower, and seed of TT was extracted using the RNA plant Plus Reagent (TIANGEN, Beijing, China) according to the manufacturer's protocol. Reverse transcription was performed using the Transcriptor First Strand cDNA Synthesis Kit (HaiGene, Harbin, China). Real-time PCR was performed on an ABI Prism 7500 sequence detector using the manufacturer's THUNDERBIRD SYBR qPCR Mix (Toyobo, Osaka, Japan). The utilized primers were: 18srRNA-F, 5'TTGACGGAAGGGCACCA-3',18srRNA-R5'-ACCACC ACCCATAGAATCAAGAA-3',4CL2-qPCR-F5'-CTCA
TCGAGCACAGCAACACTGAATTCAA-3',4CL2-qPC

R-R 5'-CTGGTACAACAGGCTTACCCAAAGGAG TC-3'. The PCR assay was performed as follows: $95^{\circ} \mathrm{C}$ for $1 \mathrm{~min}$, and 40 cycles of $95^{\circ} \mathrm{C}$ for 15 ), $60^{\circ} \mathrm{C}$ for $35 \mathrm{~s}$, and 72 ${ }^{\circ} \mathrm{C}$ for $20 \mathrm{~s}$ (Li et al., 2015). The relative expression was analyzed according to the $2^{-\Delta \Delta C T}$ method (Livak and Schmittgen, 2001).

\section{Assay of $4 C L$ enzyme activity}

The protein content of extracts from the root, stem, leaf, and flower of TT was assayed using the Coomassie brilliant blue method. The enzyme activity was assayed according to the instructions of the 4-coumaric acid: coenzyme A ligase (4CL) enzyme-linked immunoassay kit (Beijing GDELISA Biotechnology Co., Ltd., Beijing, China). The specific activities of $4 \mathrm{CL}$ were calculated as: Enzyme activity (IU) / Protein (mg).

\section{Assay of lignin content}

A qualitative study was performed using phloroglucinol- $\mathrm{HCl}$ staining. A cross section of the main tobacco stem was placed on a glass slide for immediate phloroglucinol- $\mathrm{HCl}$ staining. The sections were observed under a microscope, and then photographed.

Fresh seedlings grown for 10 days were taken and the lignin content was measured according to the method by Fukushima and Hatfield $(2001,2004)$. The absorbance value was measured at $280 \mathrm{~nm}$, with A2 $80 \mathrm{~nm} / \mathrm{mg}$ protein representing the lignin content.

Roots, stems, leaves, flowers, and seeds of tobacco were taken, and the lignin content of mature tobacco was determined according to the method described by Romualdo et al. (2001), with the absorbance value being measured at $280 \mathrm{~nm}$.

$$
\text { Lignin } \%=\frac{A b s \times \text { liters } \times 100 \%}{W_{\text {sample }} \times A_{\text {standard }}}
$$

Lignin\%: lignin content; Abs: absorbance value of the sample solution at $280 \mathrm{~nm}$, Liters: volume (L) of the sample solution, $\mathrm{W}_{\text {sample: }}$ absolute dry weight of the sample $(\mathrm{g})$, $\mathrm{A}_{\text {standard: }}$ standard absorbance of lignin in tobacco, $\mathrm{A}_{\text {standard }}=$ 20.0 .

\section{Assay of flavonoids}

Flavonoids were extracted according to the method of Burbulis et al. (1996), and the extracted samples were stored at $4{ }^{\circ} \mathrm{C}$, or retained for HPLC analysis. HPLC analysis was performed on a chromatography workstation, comprising a universal sampler (model U6K; Waters), a dual pump system (model 510; Waters, Bedford, MA), an automatic gradient controller (model 680; Waters), a photodiode array detector (model 484; Waters), and a data module (model 745B; Waters). Samples were loaded on C18 reversed-phase columns (Nova-Pak 60A, $4 \mu \mathrm{m}, 3.9$ x 75 $\mathrm{mm}$ ) at $25^{\circ} \mathrm{C}$ at a flow rate of $2.5 \mathrm{~mL} / \mathrm{min}$. The eluate was 
collected and detected at $255 \mathrm{~nm}$. Kaempferol (Sigma) dissolved at $0.1 \mathrm{mg} / \mathrm{mL}$ in $80 \%$ methanol was used as standard sample. The retention time of the samples was compared to the standard sample. The monomer concentration in the test agent was assayed via the integral of the area of the eluting peak.

\section{Statistical analysis}

All experiments were performed in at least three biological replicates, and the data were analyzed using SPSS 20.0 software.

\section{Results}

\section{Identification of $\mathrm{T}_{1}$-generation transgenic tobacco}

The expression vector pBI121-Df4CL2 was transfected into a sterile tobacco leaf by the Agrobacterium EHA105 to induce s callus, and the $\mathrm{T}_{0}$-generation of TT plants was then cultivated. $\mathrm{T}_{0}$-generation kanamycin-resistant plants were screened by PCR, and seeds were obtained from successfully transferred plants. The $\mathrm{T}_{1}$-generation of TT obtained from seeds was also screened by PCR, and the successfully transferred plants were reserved for subsequent experiments.

As shown in Figure 1, a amplicon band was found at $1700 \mathrm{bp}$, which was consistent with the predicted length of $D f 4 C L 2$. This indicates that the Df4CL2 gene was successfully transferred into tobacco.

\section{State of growth in transgenic tobacco}

The morphological characteristics and growth of the $\mathrm{T}_{1}$-generation of both TT and WT were observed at $90 \mathrm{~d}$, $120 \mathrm{~d}$, and $150 \mathrm{~d}$, respectively. As shown in Figure 2, TT was 1.7-, 1.8-, and 1.5-fold higher than WT at $90 \mathrm{~d}$ (Figure 2A), $120 \mathrm{~d}$ (Figure 2B), and $150 \mathrm{~d}$ (Figure 2C), respectively. In addition, the number of leaves was increased by

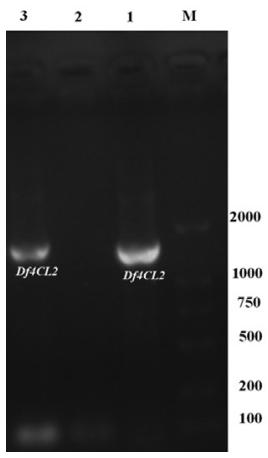

Figure 1 - PCR amplification of $D f 4 C L 2$ in $\mathrm{T}_{1}$-generation. Lanes: 1 and 3 , TT; 2, WT; M, marker.

1.3-, 1.7-, and 1.3-fold, respectively. Compared to WT, the flowering period of TT was initiated 14 days earlier.

\section{Expression of Df4CL2 in different tissues of transgenic tobacco}

The expression levels of Df4CL2 from different tissues (root, stem, leaf, flower, and seed) in TT were analyzed using fluorescent quantitative PCR with $18 \mathrm{~S}$ rRNA as internal control.

As shown in Figure 3, the expression levels of $D f 4 C L 2$ in TT can be arranged in a descending order: stem $>$ leaf $>$ root $>$ flower $>$ seed. Its expression level in the stem (which was the tissue with the highest expression) was 1.7-fold higher than in the leaf, 5.5-fold higher than in the root, 34-fold higher than in the flower, and 164-fold higher than in the seed.

\section{Analysis of $4 C L$ enzyme activity}

The protein contents in different tissues of TT and WT were assayed by the Coomassie Brilliant Blue method. As shown in Figure 4A, compared to WT, in TT the protein content was found increased at different levels. The protein concentration $(\mathrm{mg} / \mathrm{mL})$ in the root, stem, leaf, flower, and

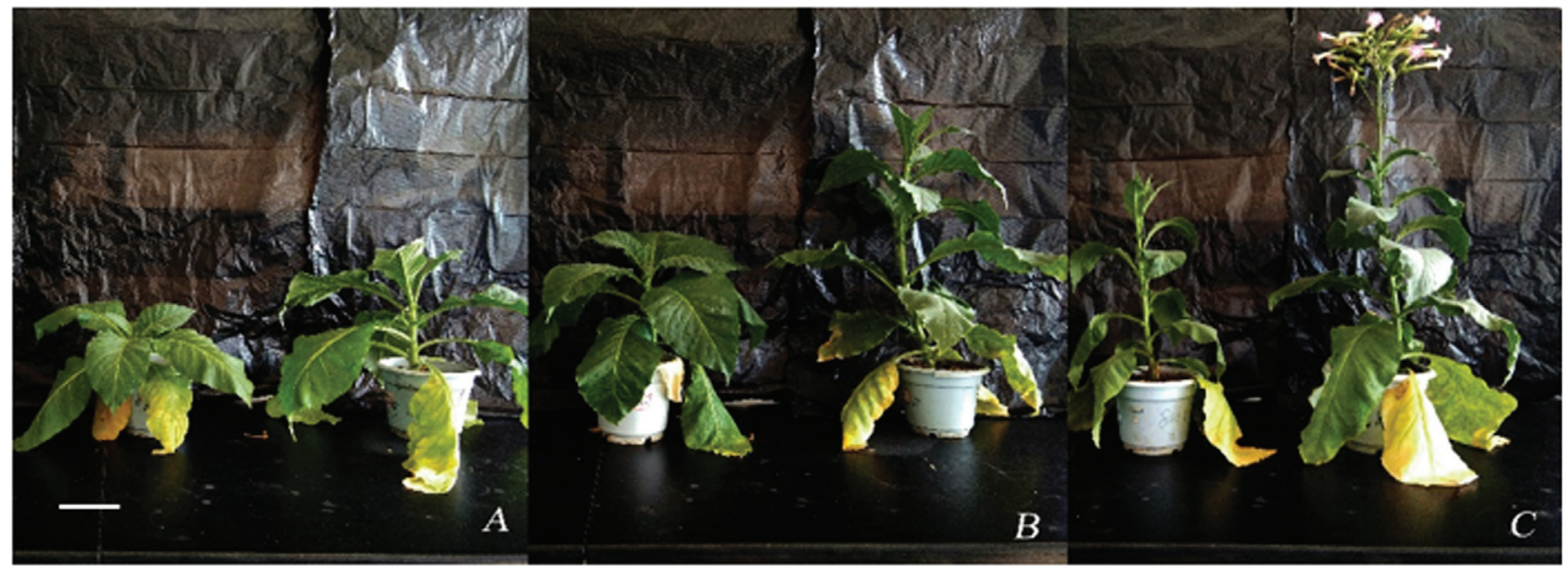

Figure 2 - Growth and development of tobacco in different periods. TT and WT grown for 90 (A), 120 (B), and 150 days (C). The left plant is WT, the right plant is TT. Bar size is $11.5 \mathrm{~cm}$. 


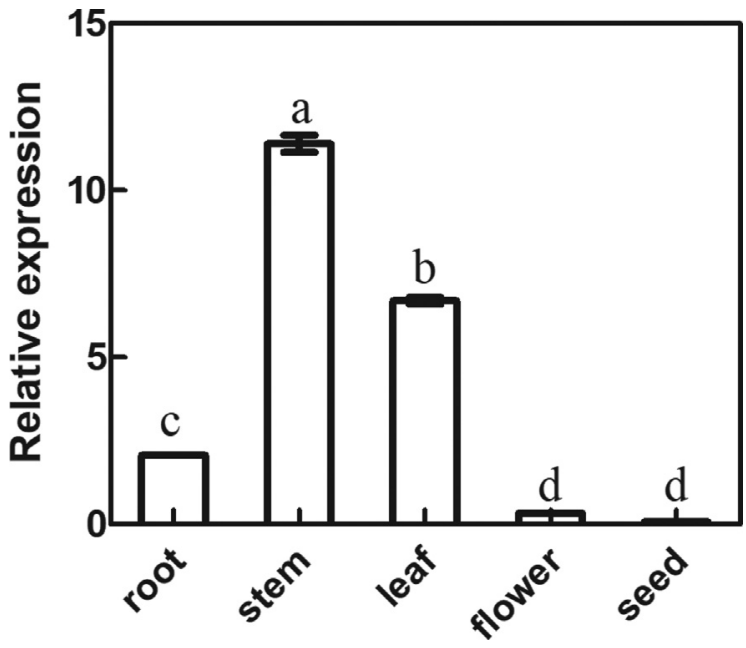

Figure 3 - Analysis of $D f 4 C L 2$ expression levels in TT using Real-time PCR and $18 \mathrm{~S}$ rRNA as internal control. Each histogram represents the mean of three biological replicates. Means and standard deviations (SD) (n $=3$ ) of at least three independent experiments are reported. Different letters indicate significant difference $(p<0.05$, one-way ANOVA, followed by Duncan's test).

seed was increased by 1.36-,1.33-, 1.48-, 1.56-, and 1.81fold, respectively.

The 4CL enzyme activities in the root, stem, leaf, flower, and seed from WT and TT were measured with the plant 4-coumaric acid: coenzyme A ligase enzyme-linked immunoassay kit. As shown in Figure 4B, the enzyme activity of $4 \mathrm{CL}$ in TT was enhanced in all tissues. Compared to WT, in TT, the enzyme activity increased by 1.8-, 1.93-, 1.81-, 1.96-, and 1.97-fold in the root, stem, leaf, flower, and seed, respectively. This increase in enzyme activity should enhance the reaction rate of the metabolic pathway and promote the synthesis of metabolites.

The specific activities in different tissues in TT and WT were also analyzed. As shown in Figure 4C, the 4CL specific activities in all TT tissues were higher than those of WT. The increase in the root, stem, leaf, flower, and seed was 1.34-, 1.40-, 1.21-, 1.25-, and 1.67-fold, respectively. A higher specific activity indicates a higher enzyme activity.
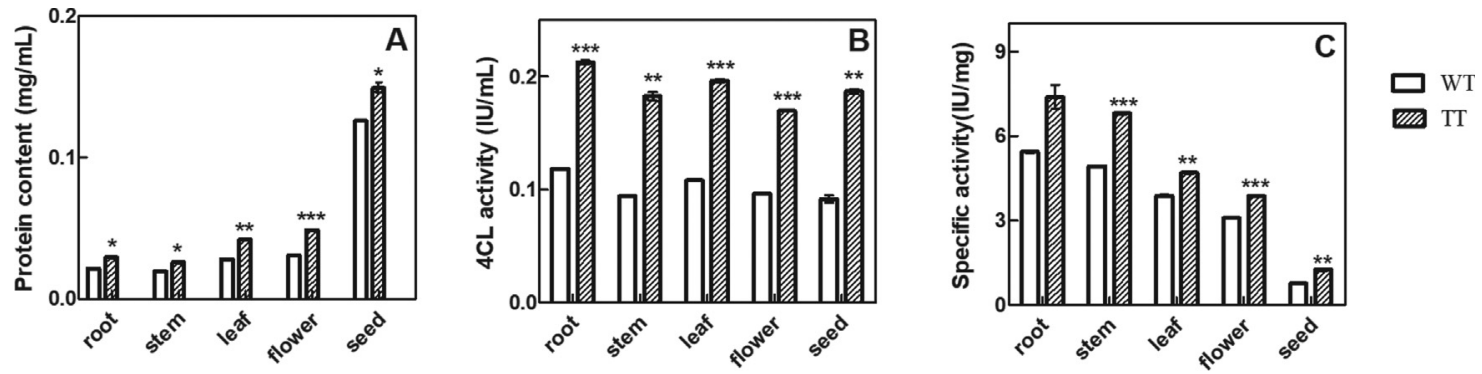

Figure 4 - Analysis of enzyme activity of 4CL in the different tissues of WT and TT. (A) Protein content; (B) enzyme activity; (C) specific activity. The values are presented as means \pm standard deviations $(\mathrm{SD})(\mathrm{n}=3) . *, * *, * * *$ indicate significant differences in comparison to the WT plants at $p<0.05, \mathrm{v}<$ 0.01 , and $p<0.001$, respectively (Student's $t$-test).

\section{Analysis of lignin content}

To test the function of $D f 4 C L 2$, the lignin content of seedlings and mature plants was analyzed and assayed by phloroglucinol staining of cross sections of the tobacco stem. As shown in Figure 5, the outer layer showed the epidermis and the cortex of the main stem. In the central part of the stele, the red part stained by phloroglucinol is the xylem. This shows that, compared to WT, the color of the TT xylem was darker, indicating that the lignin content of TT was higher.

In addition, the lignin contents in seedling and the root, stem, leaf, flower, and seed were assayed for mature WT and TT plants. As shown in Figure 6, compared to WT, the lignin content in TT seedling was increased by $1.22-$ fold. In mature plants, the root, stem, leaf, flower, and seed tissues showed an increase in lignin content by 1.25-, 1.37-, 2.0-, 1.70-, and 1.19-fold, respectively.

\section{Analysis of flavonoid content in different tissues of transgenic tobacco}

The kaempferol contents in the root, stem, leaf, flower, and seed of TT were assayed by HPLC. As shown in Figure 7, the peak time of kaempferol in the TT flower was identical to the standard sample at $2.5 \mathrm{~min}$; however, there was no peak for the root, stem, leaf, and seed at this time. Kaempferol detected in the TT flower tissue was 3-fold higher than in the WT flower (Figure 8).
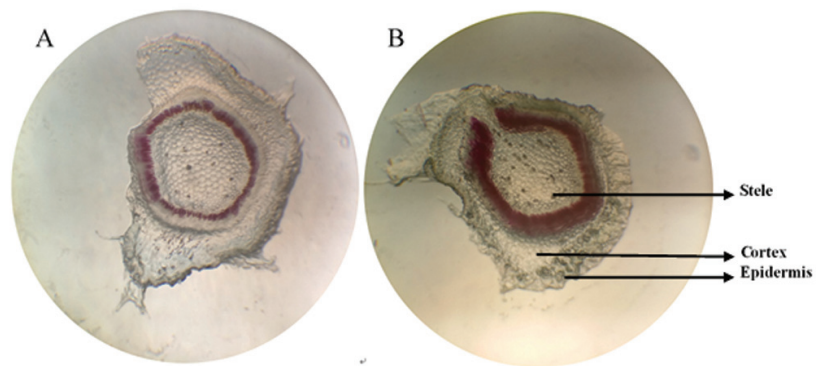

Figure 5 - Cross section of main stems from WT (A) and TT (B) seedlings, stained with phloroglucinol-HCl. The red part is the xylem of the main stem. 

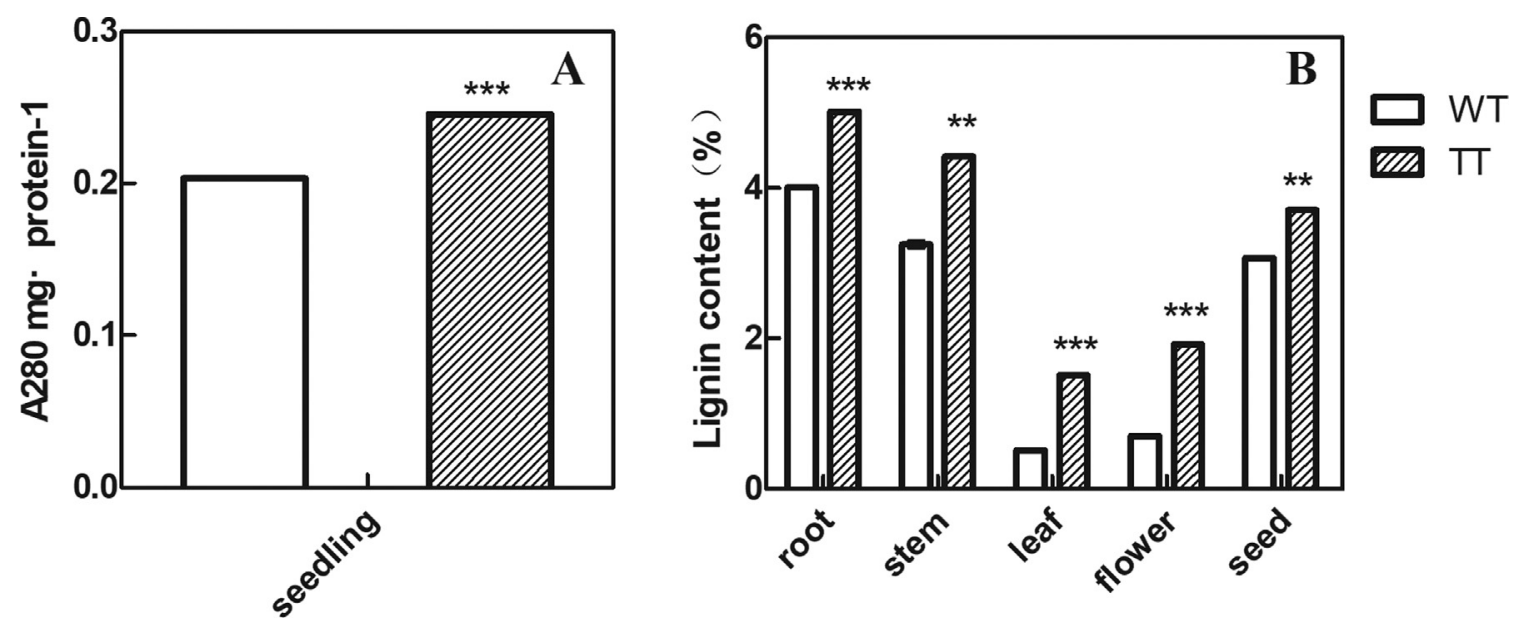

Figure 6 - Lignin content in WT and TT plants. Whole seedlings were obtained at 15 days (A), and different tissues were obtained at 120 days (B), respectively. The values and error bars indicate the mean and standard deviations $(\mathrm{SD})(\mathrm{n}=3)$, respectively, from three independent measurements. ** and *** indicate significant differences in comparison to the WT plants at $p<0.01$ and $p<0.001$, respectively (Student's $t$-test).
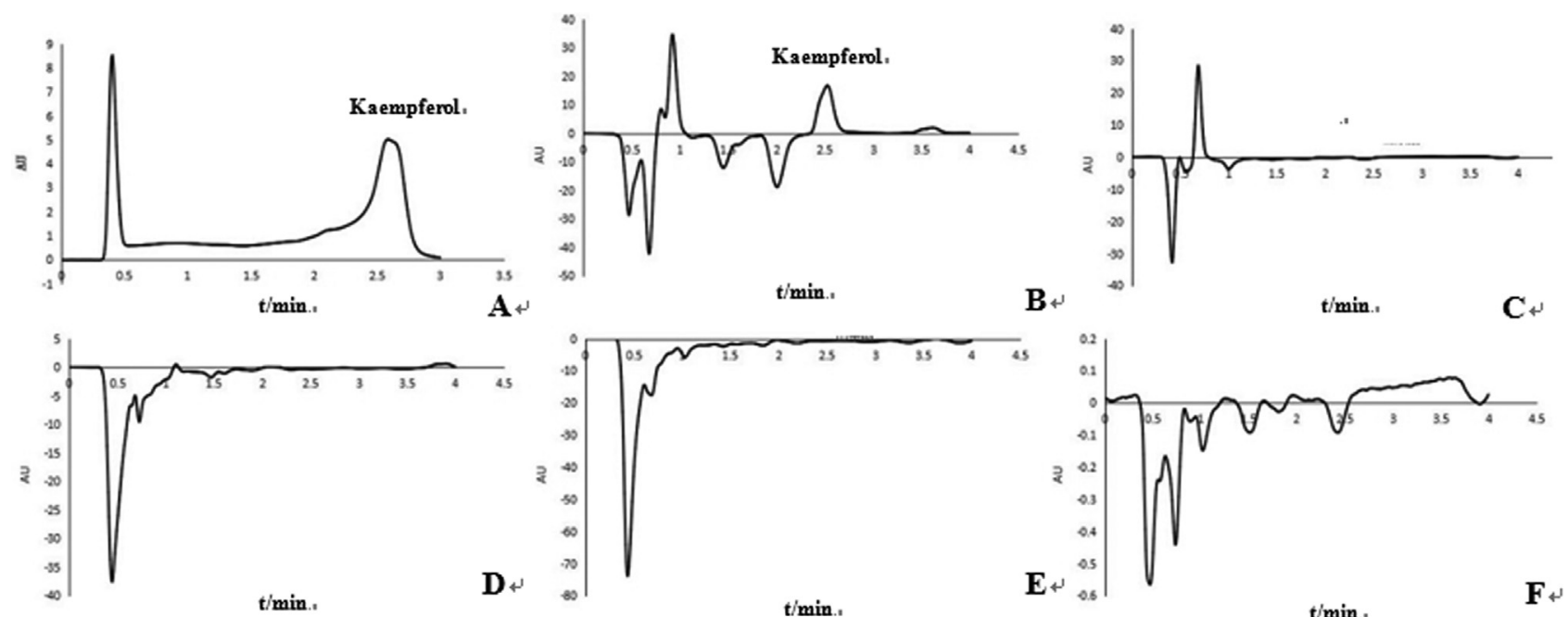

B

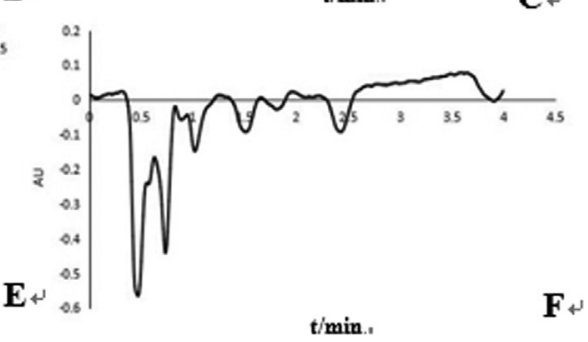

Figure 7 - Kaempferol content in TT and WT determined by HPLC. (A) Kaempferol standard; (B) flower; (C) root; (D) stem; (E) leaf; (F) seed. The peak time of kaempferol standard was $2.5 \mathrm{~min}$.

\section{Discussion}

About one fifth of the secondary metabolites in plants are produced by the phenylpropanoid metabolic pathway (Dixon et al., 2002; Hamberger and Hahlbrock, 2004). These secondary metabolites not only have multiple physiological functions in plants, but also play an important role in maintaining human health (Jorgensen et al., 2005). For example, the flavonoid rutin produced by this metabolic pathway can effectively reduce the fragility and improve the permeability of blood vessels. Therefore, this metabolic pathway has become a research focus, particularly for the production and regulation of secondary metabolites and on the regulatory mechanism of key genes.

The phenylpropanoid metabolic pathway in angiosperms has been reported in detail (Lavhale et al., 2018); however, its function in ferns remains unclear. Whether the synthesis and accumulation of these metabolites in ferns have the same or similar metabolic pathways as those of angiosperms remains to be explored. Therefore, this study investigated the key enzyme 4CL in the phenylpropanoid metabolic pathway in angiosperms and explored the relationship between Df4CL2 and metabolites in the fern $D$. fragrans.

In $D f 4 C L 2$ transgenic tobacco (TT), the expression levels of $D f 4 C L 2$ in the stem, leaf, and root were higher than in wild type (WT). In these tissues, compared to WT, the enzyme activity, as well as the lignin content was increased. This indicates that $D f 4 C L 2$ was involved in lignin synthesis. In addition, although the expression level of Df4CL2 was not high in the seed and leaf, it was found to be expressed in these tissues of TT, and compared to WT, its protein content, enzyme activity, specific activity, as well 


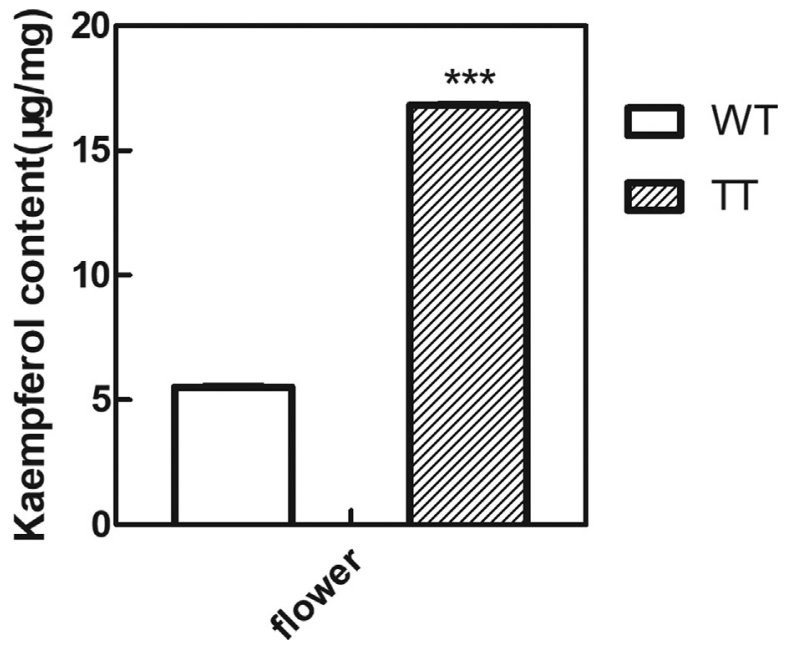

Figure 8 - Content of kaempferol in WT and TT plant flowers determined by HPLC. The values and error bars indicate the mean and standard deviations $(\mathrm{SD})(\mathrm{n}=3)$, respectively, from three independent measurements. $* * *$ indicates significant differences in comparison to the WT plants at $p<$ 0.001 , respectively (Student's $t$-test)

as the lignin content were increased. In addition, in the flower, the content of flavonoids was significantly increased compared to kaempferol used as standard sample.

From this, it can be concluded that DfACL2 is involved in the synthesis of lignin and flavonoids. However, several problems remain. For example, the expression levels of $D f 4 C L 2$ in different tissues are not consistent with the expression trends of the respective protein, enzyme activity, specific activity, and lignin content. Therefore, a literature search was conducted. First, since the genes in ferns were transferred to tobacco plants, these can express enzymatic activity. While, the enzyme activity of $4 \mathrm{CL}$ is substrate-specific, the enzyme activity of Df4CL2 in tobacco plants may be affected by the contents of different substrates in different tissues. Different substrate affinities may direct metabolic flux via different pathways for the synthesis of a variety of phenolic compounds, including different monolignols, flavonoids, isoflavonoids, coumarins, and suberin (Naoumkina et al., 2010). Secondly, in specific plants, 4CL is functionally differentiated, e.g., in Arabidopsis, At4CL3 is responsible for the synthesis of flavonoids, and At4CL1 and At4CL2 are responsible for the synthesis of lignin (Costa et al., 2003, 2005). However, $4 \mathrm{CL}$ in specific plants showed no differentiation of gene functions, which are responsible for the synthesis of lignin and flavonoids (member of 4CL gene family of $P$. patens). Based on the current data analysis, and compared to WT, overexpressed tobacco showed an increase of lignin and flavonoid contents, which indicates that the function of the gene $D f 4 C L 2$ in D. fragrans is related to the synthesis of lignin and flavonoids. Therefore, gene expression is not necessarily related to the specific metabolite content.

For O. basilicum, Rastogi et al. (2013) reported that RNAi suppression of Os4CL in leaves leads to a reduction in leaf eugenol content and trichome transcript levels. Considerable increases in endogenous 4-coumarate, ferulate, cinnamate, and caffeate were found, while the lignin content remained unaffected.

In the present study, compared to WT, the content of lignin and flavonoids in TT was increased from the measured substance content. With regard to the growth state of the plants, TT was higher than the WT plants, the stalk was thicker, the leaves were more numerous, and the biomass of TT was higher, which should help the plants to resist adverse conditions by enhancing its lodging resistance (Moura et al., 2010). The increase in lignin content is also important for bio-fibers, as well as for biofuel products (Kim et al., 2019). Kaempferol was used as an example to determine the increase in the content of flavonoids, which is crucial for ferns, and they are important substances that play a role in folk medicine.

In summary, our experiments showed that Df4CL2 is involved in the biosynthesis of lignin and flavonoids, and might not have a differentiated gene function. The biosynthesis and accumulation of flavonoids and the lignin content in D. fragrans can be promoted by enhancing the genetic expression of Df4CL2, which lays a foundation for the extensive application of $D$. fragrans in medicinal use.

\section{Acknowledgments}

This work was partially supported by the Training Plan of Young Creative Talents of Average Four-year College of Heilongjiang (No. UNPYSCT-2017154), the Youth Academic Backbone Support Program of Education Department in Heilongjiang Province of China (No. 135109316), and the basic scientific research project of higher education institutions in Heilongiiang Province of China (No. YSTSXK201885).

\section{Conflict of Interest}

The authors declare no conflict of interest.

\section{Author contributions}

Shan-Shan Li performed the research, analyzed the data and wrote the paper, Bo Li and Zhen-Zhu Zhang contributed in experiments, Shu-Li Shao revised the manuscript, Ying Chang supervised the entire study, designed the work, revised the manuscript. All authors approved the final version.

\section{References}

Blach-Olszewska Z, Jatczak B and Rak A (2008) Production of cytokines and stimulation of resistance to viral infection in human leukocytes by Scutellaria baicalensis flavones. J Interf Cytokine Res 28:571-581.

Burbulis IE, Iacobucci M and Shirley BW (1996) A null mutation in the first enzyme of flavonoid biosynthesis does not affect male fertility in Arabidopsis. Plant Cell 8:1013-1025. 
Costa MA, Collins RE, Anterola AM, Cochrane FC, Davin LB and Lewis NG (2003) An in silico assessment of gene function and organization of the phenylpropanoid pathway metabolic networks in Arabidopsis thaliana and limitations thereof. Phytochemistry 64:1097-1112.

Costa MA, Bedgar DL, Moinuddin SG, Kim KW, Cardenas CL, Cochrane FC, Shockey JM, Helms GL, Amakura Y, Takahashi $\mathrm{H}$ et al. (2005) Characterization in vitro and in vivo of the putative multigene 4-coumarate: CoA ligase network in Arabidopsis: Syringyl lignin and sinapate/sinapyl alcohol derivative formation. Phytochemistry 66:2072-2091.

Dixon RA, Achnine L, Kota P, Liu CJ, Reddy MS and Wang L (2002) The phenylpropanoid pathway and plant defence - a genomics perspective. Mol Plant Pathol 3:371-390.

Emiliani G, Fondi M, Fani R and Gribaldo S (2009) A horizontal gene transfer at the origin of phenylpropanoid metabolism: a key adaptation of plants to land. Biol Direct 16:4-7.

Fukushima RS and Hatfield RD (2001) Extraction and isolation of lignin for utilization as a standard to determine lignin concentration using the acetyl bromide spectrophotometric method. J Am Chem Soc 49:3133-3139.

Fukushima RS and Hatfield RD (2004) Comparison of the acetyl bromide spectrophotometric method with other analytical lignin methods for determining lignin concentration in forage samples. J Agric Food Chem 52:3713-3720.

Gui J, Shen J and Li L (2011) Functional characterization of evolutionarily divergent 4-coumarate: coenzyme a ligase in rice. Plant Physiol 157:574-586.

Hamberger B and Hahlbrock K (2004) The 4-coumarate: CoA ligase gene family in Arabidopsis thaliana comprises one rare, sinapate-activating and three commonly occurring isoenzymes. Proc Natl Acad Sci U S A 101:2209-2214.

Harding SA, Leshkevich J, Chiang VL and Tsai CJ (2002) Differential substrate inhibition couples kinetically distinct 4-coumarate: coenzyme A ligases with spatially distinct metabolic roles in quaking aspen. Plant Physiol 128:428-438.

Hoekema A, Hirsch PR, Hooykaas PJJ and Schilperoort RA (1983) A binary plant vector strategy based on separation of vir- and T-region of the Agrobacterium tumefaciens Tiplasmid. Nature 303:179-180.

Ito H, Muranaka T, Mori K, Jin ZX, Tokuda H, Nishino $\mathrm{H}$ and Yoshida T (2000) Ichthyotoxic phloroglucinol derivatives from Dryoperis fragrans and their anti-tumor promoting activity. Chem Pharm Bull 48:1190-1195.

Jorgensen K, Rasmussen AV, Morant M, Nielsen AH, Bjarnholt N, Zagrobelny M, Bak S and Møller BL (2005) Metabolon formation and metabolic channeling in the biosynthesis of plant natural products. Curr Opin Plant Biol 8:280-291.

Kim JY, Lee HW, Lee SM, Jae J and Park YK (2019) Overview of the recent advances in lignocellulose liquefaction for producing biofuels, bio-based materials and chemicals. Bioresour Technol 279:373-384.

Lavhale SG, Kalunke RM and Giri AP (2018) Structural, functional andevolutionary diversity of 4-coumarate-CoA ligase in plants. Planta 248:1063-1078.

Li SS, Li Y, Sun LL, Chang Y and Hu BZ (2015) Identification and expression analysis of 4-Coumarate: Coenzyme A liga- se gene family in Dryopteris fragrans. Cell Mol Biol 61:2533.

Li XJ, Fu YJ, Luo M, Wang W, Zhang L, Zhao CJ and Zu YG (2012a) Preparative separation of dryofragin and aspidin BB from Dryopteris fragrans extracts by macroporous resin column chromatography. J Pharm Biomed Anal 61:199-206.

Li XJ, Wang W, Luo M, Li CY, Zu YG, Mu PS and Fu YJ (2012b) Solvent-free microwave extraction of essential oil from Dryopteris fragrans and evaluation of antioxidant activity. Food Chem 133:437-444.

Liu XY, Wang PP, Wu YF, Cheng AX and Lou HX (2018) Cloning and functional characterization of two 4-Coumarate: CoA ligase genes from Selaginella moellendorffii. Molecules 23:595.

Livak KJ and Schmittgen TD (2001) Analysis of relative gene expression data using real-time quantitative PCR and the $2^{-\Delta \Delta \mathrm{CT}}$ method. Methods 25:402-408.

Moura JCMS, Bonine CAV, de Oliveira Fernandes Viana J, Dornelas MC and Mazzafera P (2010) Abiotic and biotic stresses and changes in the lignin content and composition in plants. J Integr Plant Biol 52:360-376.

Naoumkina MA, Zhao Q, Gallego-Giraldo L, Dai X, Zhao PX and Dixon RA (2010) Genome-wide analysis of phenylpropanoid defense pathways. Mol Plant Pathol 11:829-846.

Rao GD, Pan X, Xu F, Zhang YZ, Cao S, Jiang XN and Lu H (2015) Divergent and overlapping function of five 4-Coumarate/Coenzyme A ligases from Populus tomentosa. Plant Mol Biol Rep 33:841-854.

Rastogi S, Kumar R, Chanotiya CS, Shanker K, Gupta MM, Nagegowda DA and Shasany AK (2013) 4-Coumarate: CoA ligase partitions metabolites for eugenol biosynthesis. Plant Cell Physiol 54:1238-1252.

Romualdo SF and Ronald DH (2001) Extraction and isolation of lignin for utilization as a standard to determine lignin concentration using the acetyl bromide spectrophotometric method. J Am Chem Soc 49:3133-3139.

Shen ZB, Ma YL, Jiang W and Li WL (2005) Inhibitory activity of Dryoperis fragrans extract against fungi. Chin Tradit Herb Drugs 36:735-736.

Vassão DG, Kim KW, Davin LB and Lewis NG (2010) Lignans (neolignans) and allyl/propenyl phenols: Biogenesis, structural biology, and biological/human health considerations. In: Liu HW and Mander L (eds) Comprehensive Natural Products II. Vol 1. Elsevier, Amsterdam, pp 815-928.

Wang HR, Wang R, Xu HL and Zhang YL (2008) Analgesic effect of Dryoperis fragrans (L.) schott. J Qiqihar Med Coll 29:2443-2444.

Zhang YL, Fu HY, Zhang YY, Song QY, Xu WH and Kuang HX (2008a) Chemical components from Dryoperis fragrans and their cytotoxicity. Chin Tradit Herb Drugs 39:648-651.

Zhang YL, Zeng WM, Wang HR, Zhu L, Xu HL and Kuang HX (2008b) Study on antioxidant of lignans from Dryoperis fragrans. Chin Tradit Herb Drugs 39:343-346.

Associate Editor: Hong Luo

License information: This is an open-access article distributed under the terms of the Creative Commons Attribution License (type CC-BY), which permits unrestricted use, distribution and reproduction in any medium, provided the original article is properly cited. 